\title{
Aberrant Notch signaling in glioblastoma stem cells contributes to tumor recurrence and invasion
}

\author{
JIAN-BO YU, HAO JIANG and REN-YA ZHAN \\ Department of Neurosurgery, First Affiliated Hospital, College of Medicine, \\ Zhejiang University, Hangzhou, Zhejiang 310003, P.R. China
}

Received February 25, 2015; Accepted April 29, 2016

DOI: $10.3892 / \mathrm{mmr} .2016 .5391$

\begin{abstract}
Upregulation of the Notch signaling pathway in cancer stem cells and side population (SP) cells has a major role in maintenance, self-renewal and chemoresistance. The present study isolated a cancer stem cell-like SP accounting for $4.1 \%$ of a glioblastoma cell population using a Hoechst 33342 dye exclusion assay. In this glioblastoma SP, the expression of of Notch1 signaling proteins Notch1 intracellular domain and Hes-1 was markedly upregulated. Furthermore, knockdown of Notch1 by RNA interference significantly diminished the neurosphere formation ability, self-renewal and chemoresistance of the SP cells. In addition, the expression of the stem-cell surface genes Oct-4, Sox 2 and Nanog in SP cells was significantly reduced and the sensitivity to the SP cells to chemotherapeutics was enhanced following Notch1 knockdown. In conclusion, the results of the present study suggested that upregulation of Notch1 is involved in the chemotherapy resistance and tumor recurrence of glioblastoma. Hence, the development of novel anti-cancer drugs targeting the Notch1 signaling pathway may be a promising strategy for curing glioblastoma.
\end{abstract}

\section{Introduction}

Glioblasoma multiforme (GBM) is one of the most aggressive types of malignant brain tumor and has a poor prognosis, with a median survival time of $\sim 14.6$ months and a World Health Organization (WHO) grade IV (1-3). According to the cancer stem cell theory, cancer stem cells (CSCs) and stem-like side populations (SPs) are responsible for therapy failure and tumor recurrence. These CSCs resist current conventional treatment strategies and re-initiate cancer growth; they are

Correspondence to: Mr. Ren-Ya Zhan, Department of Neurosurgery, First Affiliated Hospital, College of Medicine, Zhejiang University, 79 Qingchun Road, Hangzhou, Zhejiang 310003, P.R. China

E-mail: zhanrenya79@gmail.com

Key words: cancer stem cells, Notch1, tumor recurrence, adenosine triphosphate-binding cassette transporters, anti-cancer drug thus responsible for minimal residual disease (4-6). Several studies reported the existence of brain cancer stem cells (BCSCs), which have marked self-renewing properties owing to their elevated expression of stem-cell surface markers, high differentiation capacity, evasion of apoptosis and resistance to chemotherapeutics $(7,8)$. Therefore, understanding the molecular mechanism of BCSC-mediated tumor recurrence may aid in the development of novel cancer therapeutic drugs.

Brain cancer stem cells can be isolated using a fluorescence-activated cells sorting (FACs)-based Hoechst 33342 dye exclusion assay. The purified BCSCs are termed as SP cells, as they appear as distinct populations on the side of the main cell population in the flow cytometry dot plot $(9,10)$. Brain cancer SP cells possess the distinct features of BCSCs, including resistance to chemotherapeutics, enhanced neurosphere formation, overexpression of stem-cell surface genes, including Nanog, Oct-4, CD133, CD44, CD34, CD29 and CD24, as well as adenosine triphosphate-binding cassette $(\mathrm{ABC})$ transporter genes, including ABCB1 (MDR1), ABCC1 and ABCG2, and genes associated with resistance to apoptosis (11). In addition, Notch1 signaling was shown to be aberrantly regulated in SP cells and Notch1 inactivation enhanced their sensitivity to chemotherapeutic drug treatments $(12,13)$. Hence, elucidating the mechanism underlying Notch1 signaling-mediated drug resistance and tumor relapse will aid in the development of novel treatments targeting BCSCs and may allow the complete eradication of brain tumors. For this purpose, the present study evaluated the expression of Notch1 signaling proteins in glioblastoma SP cells isolated by FACS. Furthermore, Notch1-specific RNA interference (RNAi) technology was used to silence Notch1 in SP cells and the effects on the neurosphere formation ability and chemotherapeutic resistance of SP cells were assessed. The present study suggested that Notch1 inhibition may represent a promising strategy for the eradication of glioblastoma by targeting their SP cells.

\section{Materials and methods}

Patient samples and establishment of glioblastoma cell culture. The present study was approved by the Ethics Committee of the College of Medicine at Zhejiang University (Hangzhou, China). Written consent was obtained from all patients. Glioblastoma samples (stage, malignant glioblastoma) were collected from 10 cancer patients (age, 18-39 years; 
6 male and 4 female) undergoing surgery at the Department of Neurosurgery of the First Affiliated Hospital of Zhejiang University between January 2012 and December 2014. The samples were cut and fixed in $4 \%$ formalin solution (Merck-Millipore, Darmstadt, Germany). Fixed tissues were dehydrated in ethanol (Merck-Millipore), cleared using xylene (Merck-Millipore) and embedded in paraffin (Thermo Fisher Scientific, Inc., Waltham, MA, USA). Tissue paraffin blocks were sectioned into 5- $\mu \mathrm{m}$ slices using a microtome. A histological analysis confirmed that the patients had WHO grade IV glioblastoma.

The glioblastoma tissues harvested from the patients were mechanically disaggregated using a scalpel in Dulbecco's modified Eagle's medium: Nutrient mixture F-12 (DMEM/F-12; Mediatech, Manassas, VA, USA) and passed through $100-\mu \mathrm{m}$ nylon mesh cell strainers (Falcon; BD Biosciences, Franklin Lakes, NJ, USA). The resulting cell suspensions were washed two times using DMEM/F-12, followed by culture in complete media (DMEM/F-12) containing B27 (Invitrogen; Thermo Fisher Scientific, Inc.) plus $20 \mathrm{ng} / \mathrm{ml}$ basic fibroblast growth factor (Sigma-Aldrich, St. Louis, MO, USA), $20 \mathrm{ng} / \mathrm{ml}$ epidermal growth factor (Sigma-Aldrich), $50 \mathrm{U} / \mathrm{ml}$ penicillin and $50 \mathrm{mg} / \mathrm{ml}$ streptomycin (both Thermo Fisher Scientific, Inc.).

Isolation of SP cells. Hoechst 33342 staining and FACS were used to purify SP cells. Cultured glioblastoma cells were suspended at $10^{6} \mathrm{cells} / \mathrm{ml}$ in DMEM containing $10 \%$ fetal bovine serum (FBS; Sigma-Aldrich) and labeled with Hoechst 33342-bis-benzimide (5 $\mu 1 / \mathrm{ml}$; Sigma-Aldrich) alone $(\mathrm{n}=7)$ or in combination with verapamil $(0.8 \mu \mathrm{l} / \mathrm{ml}$; Sigma-Aldrich) ( $\mathrm{n}=7)$. Following mixing, the cell suspensions were incubated in a water bath at $37^{\circ} \mathrm{C}$ for $90 \mathrm{~min}$. Cells were then centrifuged at $65 \mathrm{xg}$ for $10 \mathrm{~min}$ at $4^{\circ} \mathrm{C}$, and then re-suspended in $500 \mu 1$ Hank's balanced salt solution containing $10 \mathrm{mM}$ 4-(2-hydroxyethyl)-1-piperazineethanesulfonic acid (Sigma-Aldrich). Finally, cells were counterstained with $2 \mu \mathrm{g} / \mathrm{ml}$ propidium iodide (Sigma-Aldrich) at $4^{\circ} \mathrm{C}$. The cells were filtered through a $50-\mu \mathrm{m}$ nylon mesh (BD Biosciences) to remove any cell clumps and were then subjected to FACS (FACS Aria II; BD Biosciences) with excitation of the Hoechst 33342 dye at $355 \mathrm{~nm}$ and analysis of its dual-wavelength fluorescence at $450 \mathrm{~nm}$ (blue) and $675 \mathrm{~nm}$ (red). SP and non-SP cells were isolated under sterile conditions and kept in DMEM containing 10\% FBS.

Glioma sphere formation assay. The gliosphere formation assay was performed according to the procedure of a previous study (14). After seven days of culture, the numbers of spheres generated from SP and non-Sp cells were counted. Three independent experiments were performed.

Reverse transcription-quantitative polymerase chain reaction $(R T-q P C R)$. Total RNA was extracted and reverse-transcribed into cDNA using a Reverse Transcriptase kit (Fermentas; Thermo Fisher Scientific, Inc.). An iCycler IQ real-time detection system (Bio-Rad Laboratories, Inc., Hercules, CA, USA) using IQ Supermix with SYBR-Green (Bio-Rad Laboratories, Inc.) was used for qPCR analysis. The primer sequences for Notch1 and Hes-1, as well as the reaction conditions, were designed based on a previous study (13) and were as follows: Notch1 forward, 5'-GCGAGGTCAACA CAGACGAG-3' and reverse, 5'-CAGGCACTTGGCACC ATTC-3'; and Hes-1 forward, 5'-TGGAGAGGCGGCTAA GGTGT-3' and reverse, 5'-GCTGGTGTAGACGGGGAT GAC- 3 '. The primer sequences for the other genes were as follows, according to a previous study (15): ABCG2 forward, 5'-GGATGAGCCTACAACTGGCTT-3' and reverse, 5'-CTT CCTGAGGCCAATAAGGTG-3'; Oct4 forward, 5'-TCGAGA ACCGAGTGAGAGGC-3' and reverse, 5'-CACACTCGG ACCACATCCTTC-3'; Sox 2 forward, 5'-CACACTGCCCCT CTCACACAT-3' and reverse, 5'-CATTTCCCTCGTTTTTCT TTGAA-3'; Nanog forward, 5'-CCAACATCCTGAACCTCA GCTAC-3' and reverse, 5'-GCCTTCTGCGTCACACCATT-3'; and GAPDH forward, 5'-TCTGCTCCTCCTGTTCGACA-3' and reverse, 5'-AAAAGCAGCCCTGGTGACC-3'. The thermocycling conditions were as follows: Initial denaturation at $94^{\circ} \mathrm{C}$ for $2 \mathrm{~min}$, followed by 35 cycles of denaturation at $-95^{\circ} \mathrm{C}$ for $15 \mathrm{sec}$, annealing at $-58^{\circ} \mathrm{C}$ for $45 \mathrm{sec}$ and extension at $-60^{\circ} \mathrm{C}$ for $30-45 \mathrm{sec}$. The final extension step occurred at $72^{\circ} \mathrm{C}$ for $5 \mathrm{~min}$. The mRNA expression levels were normalized to the GAPDH internal reference gene and the relative expression levels were calculated using the $2^{-\Delta \Delta C q}$ method (16). Reactions were performed in triplicate.

Transfection of small interfering (si)RNAs. siRNAs specific to Notch1 were purchased from Dharmacon (Lafayette, CO, USA), and had the following sequences: Notch1\#1, 5'-GCC UGGACAAGAUCAAUGAtt-3'; and Notch1\#2, 5'-GCCUGU CUGAGGUCAAUGAtt-3'. Scrambled siRNAs (Dharmacon) containing the same nucleotide content as the Notch1 siRNAs, but in a random sequence, were used as the control. SP cells were transfected with $1 \mu \mathrm{g} / \mu \mathrm{l}$ siRNAs using Lipofectamine ${ }^{\circledR} 2000$ (Invitrogen; Thermo Fisher Scientific, Inc.), according to the manufacturer's instructions. The siRNA-transfected SP cells were subjected to the assays after $48 \mathrm{~h}$ of incubation.

Western blot analysis. Total protein was extracted from the SP and non-SP cells, and the protein concentration was determined using the Bradford Protein assay (Sigma-Aldrich). Equal quantities of protein ( $20 \mu \mathrm{g} / \mathrm{lane}$ ) were subjected to $10 \%$ SDS-PAGE and transferred onto nitrocellulose membranes. The membranes were then blocked using Tris-buffered saline supplemented with $0.1 \%$ Tween-20 (TBST; $50 \mathrm{mM}$ Tris-HCl, $\mathrm{pH} 8.2,150 \mathrm{mM} \mathrm{NaCl}$ ) containing $5 \% \mathrm{w} / \mathrm{v}$ non-fat dry milk and subsequently incubated with the rabbit anti-Notch1 monoclonal antibody (1:5,000; ab52627; Abcam, Cambridge, UK), rabbit anti-ABCG2 polyclonal antibody $(1: 10,000$; ab63907; Abcam), rabbit anti-Hes-1 polyclonal antibody (1:500; ab71559; Abcam) and rabbit anti-GAPDH polyclonal antibody (1:10,000; ab37168; Abcam) for $3 \mathrm{~h}$ at room temperature. GAPDH was used as a loading control. Following washing three times for 10 min each with $1 \mathrm{X}$ TBST, the cells were treated with alkaline phosphatase-conjugated goat anti-rabbit immunoglobulin $\mathrm{G}$ heavy\&light chains $(1: 5,000$; ab97048; Abcam) for $3 \mathrm{~h}$ at room temperature. Antibodies were visualized using the Pierce ${ }^{\mathrm{TM}}$ ECL Western Blotting Substrate (Thermo Fisher Scientific, Inc.). Subsequently, blots were scanned and quantitative analysis was performed using a densitometer (Bio-Rad GS-710; Bio-Rad Laboratories, Inc.). 

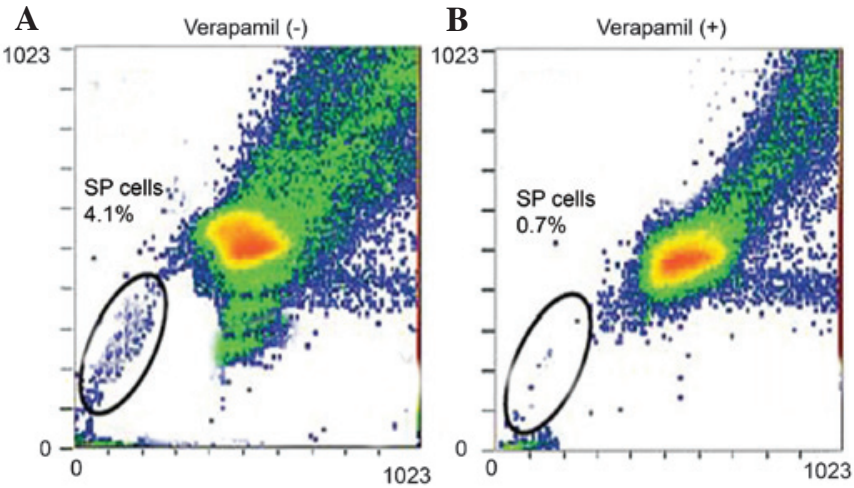

Figure 1. Purification of cancer stem-like SP cells by fluorescence-assisted cell sorting using a Hoechst 33342 dye exclusion assay. (A) Untreated human glioblastoma samples with a SP of $4.1 \%$ (gated region). (B) Treatment with adenosine triphosphate-binding cassette transporter inhibitor verapamil reduced the SP to $0.7 \%$ (gated region). Cells were counter-stained with propidium iodide. SP, side population.

$\mathbf{A}$

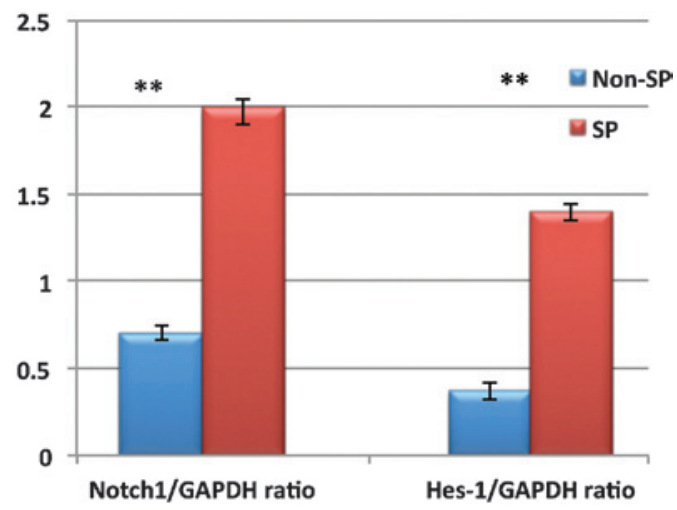

B

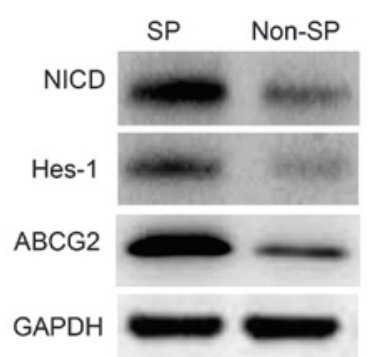

Figure 2. Evaluation of Notch signaling proteins in SP and non-SP cells. (A) Reverse-transcription quantitative polymerase chain reaction analysis showed that the expression of Notch1 and Hes-1 was significantly upregulated in SP cells compared with that in non-SP cells. Values are expressed as the mean \pm standard deviation. ${ }^{* *} \mathrm{P}<0.01$ vs. the non-SP cells. (B) Western blot analysis indicated increased protein expression of NCID and Hes-1 in SP cells compared with that in non-SP cells. GAPDH was used as a loading control. SP, side population; NCID, Notch1 intracellular domain.

Cell resistance assay. Cells were seeded into 96-well plates at $1 \times 10^{3}$ cells/well and incubated at $37^{\circ} \mathrm{C}$ for $24 \mathrm{~h}$, prior to treatment with the following chemotherapeutic drugs for $48 \mathrm{~h}$ : 5-Fluorouracil (5-FU; $10 \mu \mathrm{g} / \mathrm{ml})$, oxaliplatin $(100 \mathrm{mM})$ and cisplatin ( $5 \mathrm{mg} / \mathrm{ml}$; all Sigma-Aldrich). Cell resistance was determined by calculating the mean optical density (OD) at $450 \mathrm{~nm}\left(\mathrm{OD}_{450}\right)$ for three independent experiments for each group. The resistance of the groups to the chemotherapeutic drugs was calculated using the following formula: Resistance rate $(\%)=\left[\mathrm{OD}_{450 \text { (experimental group) }} / \mathrm{OD}_{450 \text { (control group) }}\right] \times 100$.

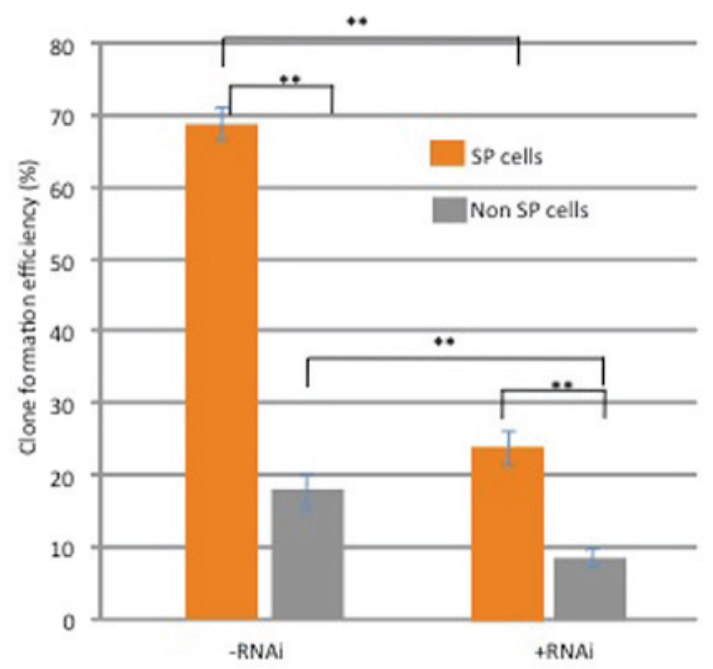

Figure 3. Notch1 depletion reduces the neurosphere formation efficiency of $\mathrm{SP}$ cells. The total number of tumor spheres generated from glioblastoma SP cells purified by fluorescence-activated cell sorting was significantly higher than that generated from non-SP cells. After Notch1-specific RNA interference, the generation of neurospheres by SP cells and non-SP cells was significantly reduced. Values are expressed as the mean \pm standard deviation of three independent experiments. ${ }^{* *} \mathrm{P}<0.01$. SP, side population.

Statistical analysis. Data are expressed as the mean \pm standard deviation. Statistical analyses were performed using GraphPad Prism 5.0 software (GraphPad Software, Inc., La Jolla, CA, USA). One-way analysis of variance and Student's $t$-test were performed to assess significant differences between experimental and control groups. $\mathrm{P}<0.05$ was considered to indicate a statistically significant difference between values.

\section{Results}

Notch signaling is upregulated in glioblastoma SP cells. Staining with the cell-permeable DNA-binding dye Hoechst 33342 and FACS analysis showed that untreated glioblastoma cells contained a SP of $\sim 4.1 \%$ (Fig. 1A). Furthermore, following treatment with the ABS transporter inhibitor verapamil, the SP was diminished to $0.7 \%$ (Fig. 1B). Subsequently, the SP cells isolated by FACS were subjected western blot and RT-qPCR analysis of proteins and mRNA involved in Notch signaling pathways, including NICD and Hes-1. NICD and Hes-1 expression was significantly upregulated in the SP cells when compared to that in the non-SP cells (Fig. 2A and B). These results confirmed the presence of SP cells in glioblastoma and indicate that Notch signaling is upregulated in SP cells.

Elevated Notch signaling enhances the self-renewal capacity of glioblastoma SP cells. In order to investigate the role of Notch signaling in the self-renewal of SP cells, the effects of Notch1 RNAi on their sphere forming ability were analyzed. As shown in Fig. 3, the ability of native SP cells to form tumor spheres was significantly enhanced, as compared with non-SP cells $(\mathrm{P}<0.01)$. However, the sphere forming capacity of SP cells was significantly compromised following RNAi of Notch1 $(\mathrm{P}<0.05$; Fig. 3). In addition, the expression levels of the stem cell-surface proteins Oct-4, Nanog and Sox 2 were assessed. As shown in Fig. 4, the relative mRNA expression levels of these 


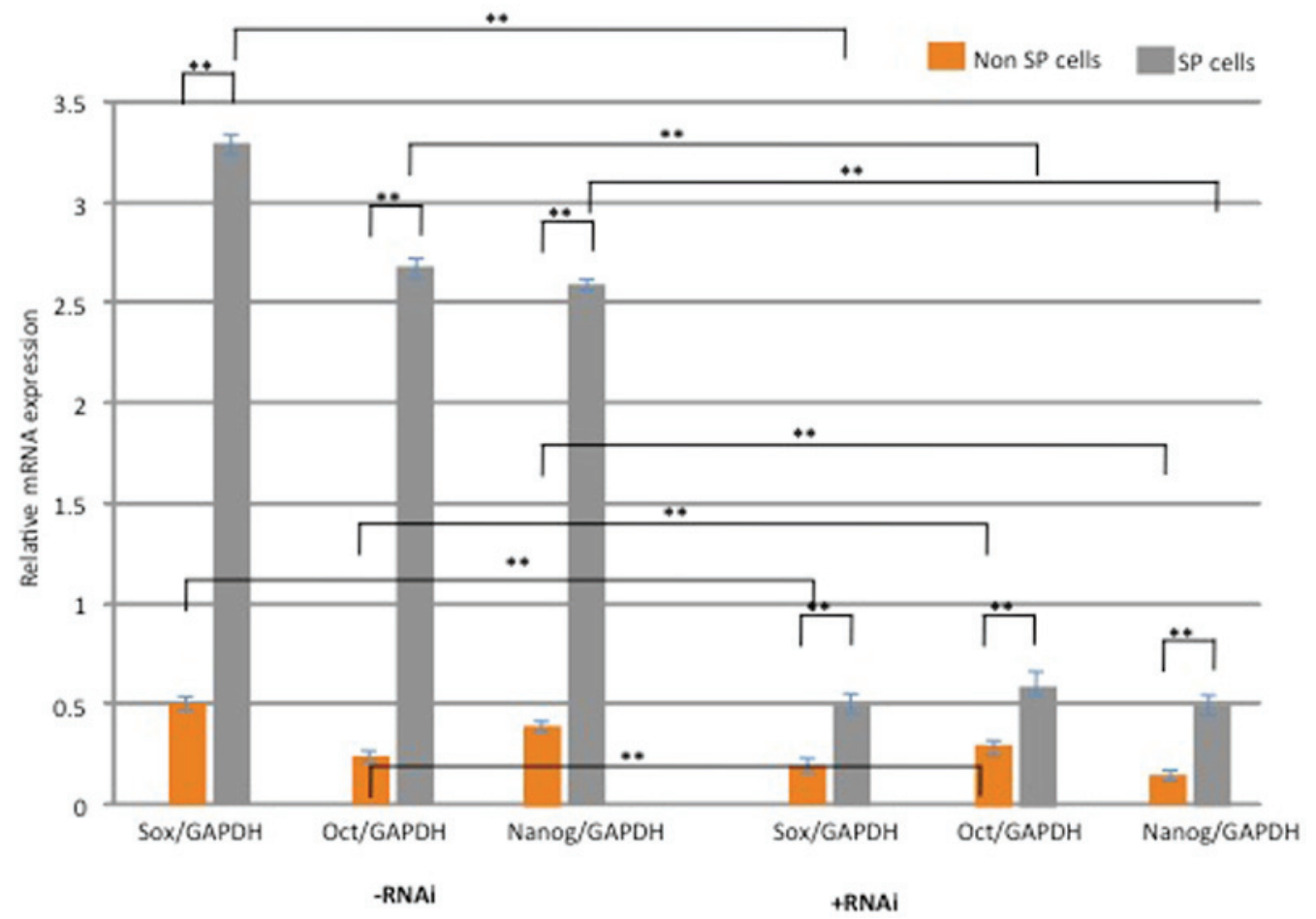

Figure 4. Depletion of Notch1 led to the downregulation of stemness genes in SP cells. Reverse-transcription polymerase chain reaction analysis showed that the relative mRNA expression levels of Oct-4, Sox2 and Nanog were significantly higher in SP cells than that in non-SP cells. Notch1-specific RNA interference markedly reduced the mRNA expression levels of Oct-4, Sox 2 and Nanog. Values are expressed as the mean \pm standard deviation. ${ }^{* *} \mathrm{P}<0.01$. SP, side population.

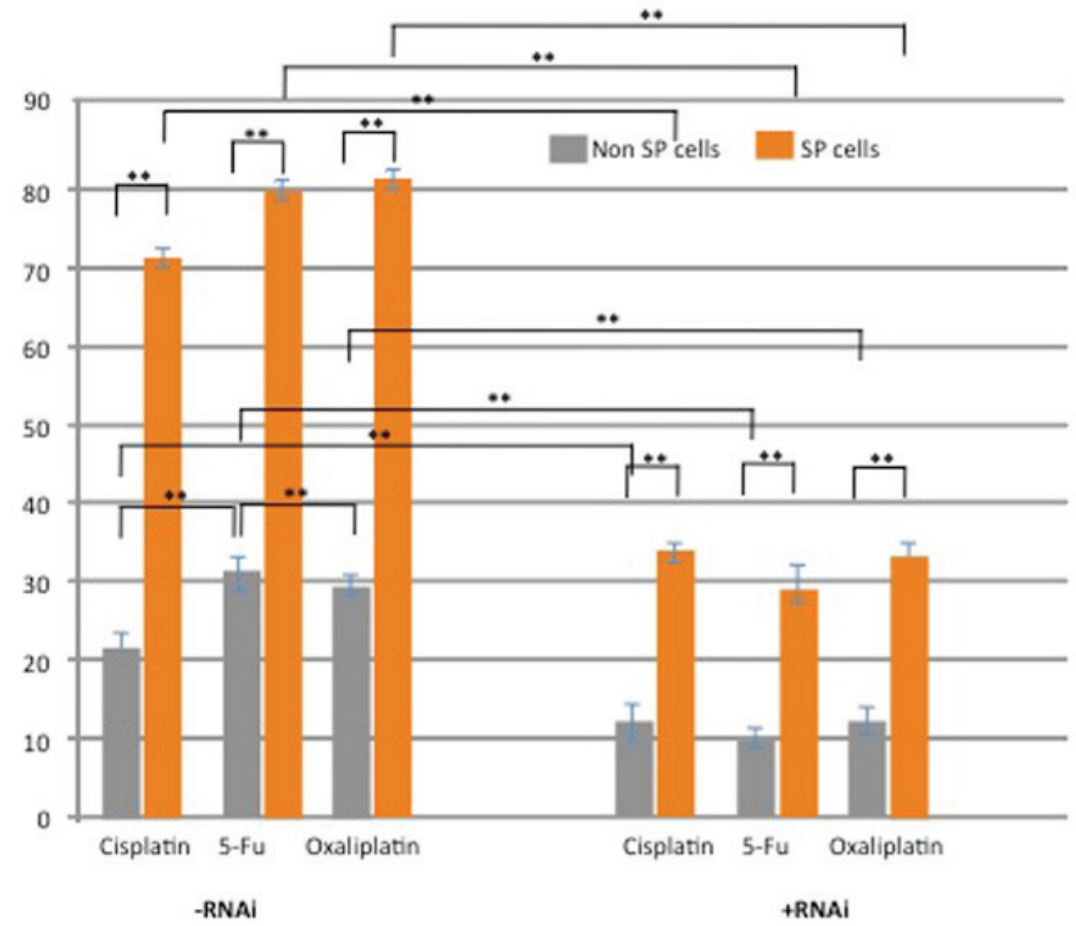

Figure 5. Depletion of Notch1 enhances the sensitivity of SP cells to DNA-targeting drugs. SP cells showed an enhanced cell survival rate compared with that of non-SP cells following treatment with 5-FU, cisplatin and oxaliplatin. Silencing of Notch1 in SP cells enhanced the susceptibility of SP cells to the drugs and significantly reduced the cell viability of SP cells. Values are expressed as the mean \pm standard deviation. ${ }^{* *} \mathrm{P}<0.01$. SP, side population; 5-FU, 5-fluorouracil.

genes were significantly upregulated in SP cells, as compared with non-SP cells $(\mathrm{P}<0.05)$. However, following knockdown of Notch1, the expression of these genes was significantly down- regulated in SP cells $(\mathrm{P}<0.05$; Fig. 4). These results suggest that activation of Notch1 in glioblastoma SP cells may have a crucial role in the maintenance and self-renewal of SP cells. 
Overexpression of Notchl is associated with resistance of SP cells to chemotherapy. Next, the present study performed a chemotherapy resistance assay in order to determine the survival rates of SP and non-SP cells following treatment with the DNA-targeting drugs 5-FU, cisplatin and oxaliplatin. The cell survival rate of SP cells treated with the chemotherapeutics was significantly higher than that of non-SP cells following treatment $(\mathrm{P}<0.05)$ (Fig. 5). However, knockdown of Notch1 in SP cells significantly reduced the survival rate of SP cells following incubation with the drugs (Fig. 5). These results indicated that elevated Notch1 signaling may be associated with chemoresistance and evasion of apoptosis of glioblastoma SP cells.

\section{Discussion}

According to the CSCs theory, the resistance of small populations of CSCs or CSC-like SPs to existing chemotherapeutics, and their ability to re-initiate tumors following chemotherapy, are the main reasons for tumor recurrence and metastasis following treatment (4-6). Therefore, the targeting of CSCs is required in order to provide effective cancer treatments and to improve the overall survival rate of patients with cancer.

The present study aimed to characterize a CSC-like SP of glioblastoma and to evaluate the role of Notch1 signaling in their tumorigenic and chemoresistant phenotypes. Previous studies on a glioblastoma cell line detected the existence of 1-2.5\% CSC-like SP cells, which possessed all of the characteristic features of CSCs. $(3,17)$. In the present study, FACS was used to identify and isolate a CSC-like SP of $\sim 4.1 \%$ from patient-derived glioma cells. Upon treatment with the ABC transporter inhibitor verapamil, the SP was markedly reduced to $0.7 \%$. At high concentrations, verapamil specifically inhibits ABCG2 transporter proteins (18); thus suggesting that the upregulation of ABCG2 transporter proteins may be involved in the resistance of glioblastoma SP cells to therapeutic drugs.

A previous study reported that elevated expression of ABCG2 was involved in downstream targeting of Notch1 signaling, which promoted the expression of stem cell-associated proteins such as Nestin in glioma cells (19). Notch1 signaling was shown to efficiently promote the proliferation and self-renewal of SP cells $(20,21)$. Therefore, the present study analyzed Notch1 signaling in patient-derived glioblastoma SP cells isolated by FACS. In the SP cells, the expression of Notch1 signaling genes NICD and Hes-1 at the protein and mRNA levels were markedly upregulated, as compared with that in non-SP cells. Furthermore, the SP cells showed enhanced drug resistance and elevated expression of stem-cell surface genes Oct-4, Sox-2 and Nanog, which may be major factors in the maintenance and self-renewal, which was also shown to be enhanced in SP cells compared with that of non-SP cells. Of note, silencing of Notch1 signaling reduced the self-renewal capacity, enhanced the sensitivity of SP cells to DNA-targeting chemotherapeutic drugs and the expression of stemness genes was also significantly downregulated. Consistent with these findings, previous studies on different cancer types demonstrated that Notch-depleted SP cells were more sensitive to chemotherapy and apoptosis, and that their self-renewal ability and high proliferation rate were also compromised (22-25). All of these results suggested that Notch1 signaling has a crucial role in drug resistance and maintenance of self-renewal of SP cells.

In conclusion, the present study suggested that elevated Notch1 signaling in glioblastoma SP cells is responsible for drug resistance and recurrence of glioblastoma. However, the link between Notch1 signaling and cell division/cell death signaling mechanisms requires to be further elucidated in order to facilitate the development of novel anti-cancer drugs to target CSCs and possibly cure glioblastoma.

\section{Acknowledgements}

The authors would like to thank Dr Dong Chen (Department of Neurosurgery, Tianjin Huanhu Hospital, Tianjin, China) and Dr Tie-Jun Wang (Department of Radiation Oncology, The Second Hospital of Jilin University, Changchun, China) for sharing their experimental protocols.

\section{References}

1. Holland EC: Gliomagenesis: Genetic alterations and mouse models. Nat Rev Genet 2: 120-129, 2001.

2. Kleihues P, Louis DN, Scheithauer BW, Rorke LB, Reifenberger G, Burger PC and Cavenee WK: The WHO classification of tumors of the nervous system. J Neuropathol Exp Neurol 61: 215-225, 2002.

3. Fukaya R, Ohta S, Yamaguchi M, Fujii H, Kawakami Y, Kawase $\mathrm{T}$ and Toda $\mathrm{M}$ : Isolation of cancer stem-like cells from a side population of a human glioblastoma cell line, SK-MG-1. Cancer Lett 28: 150-157, 2010.

4. Hirschmann-Jax C, Foster AE, Wulf GG, Fujii H, Kawakami Y, Kawase T and Toda M: A distinct 'side population' of cells with high drug efflux capacity in human tumor cells. Proc Natl Acad Sci USA 101: 14228-14233, 2004.

5. Challen GA and Little MH: A side order of stem cells: The SP phenotype. Stem Cells 24: 3-12, 2006.

6. Ramachandran C and Melnick SJ: Multidrug resistance in human tumors-molecular diagnosis and clinical significance. Mol Diagn 4: 81-94, 1999.

7. Singh SK, Clarke ID, Terasaki M, Bonn VE, Hawkins C, Squire J and Dirks PB: Identification of a cancer stem cell in human brain tumors. Cancer Res 63: 5821-5828, 2003.

8. Singh SK, Hawkins C, Clarke ID, Squire JA, Bayani J, Hide T, Henkelman RM, Cusimano MD and Dirks PB: Identification of human brain tumour initiating cells. Nature 432: 396-401, 2004.

9. Kondo T, Setoguchi T and Taga T: Persistence of a small subpopulation of cancer stem-like cells in the C6 glioma cell line. Proc Natl Acad Sci USA 101: 781-786, 2004.

10. Harris MA, Yang H, Low BE, Mukherjee J, Guha A, Bronson RT, Shultz LD, Israel MA and Yun K: Cancer stem cells are enriched in the side population cells in a mouse model of glioma. Cancer Res 68: 10051-10059, 2008.

11. Robey RW, Shukla S, Finley EM, Oldham RK, Barnett D, Ambudkar SV, Fojo T and Bates SE: Inhibition of P-glycoprotein $(\mathrm{ABCB} 1)$ and multidrug resistance associated protein-1 (ABCC1)-mediate transport by the orally administered inhibitor CBT-1 (R). Biochemical Pharmacol 75: 1302-1312, 2008.

12. Phillips TM, McBride WH and Pajonk F: The response of CD24 (-/low)/CD44+ breast cancer-initiating cells to radiation. J Natl Cancer Inst 98: 1777-1785, 2006.

13. Yu S, Zhang R, Liu F, Wang H, Wu J and Wang Y: Notch inhibition suppresses nasopharyngeal carcinoma by depleting cancer stem like side population cells. Oncol Rep 28: 561-566, 2012.

14. Chearwae W and Bright JJ: PPAR $\gamma$ agonists inhibit growth and expansion of $\mathrm{CD} 33^{+}$brain tumour stem cells. Br J Cancer 99: 2044-2053, 2008.

15. Guo D, Xu BL, Zhang XH and Dong MM: Cancer stem-like side population cells in the human nasopharyngeal carcinoma cell line cne- 2 possess epithelial mesenchymal transition properties in association with metastasis. Oncol Rep 28: 241-247, 2012. 
16. Livak KJ and Schmittgen TD: Analysis of relative gene expression data using real-time quantitative PCR and the 2(-Delta Delta C(T)) Method. Methods 25: 402-408, 2001.

17. Patrawala L, Calhoun T, Schneider-Broussard R, Zhou J, Claypool K and Tang DG: Side population is enriched in tumorigenic, stem-like cancer cells, whereas ABCG2+ and ABCG2-cancer cells are similarly tumorigenic. Cancer Res 65: 6207-6219, 2005.

18. Ozvegy-Laczka C, Hegedus T, Várady G, Ujhelly O, Schuetz JD, Váradi A, Kéri G, Orfi L, Német K and Sarkadi B: High-affinity interaction of tyrosine kinase inhibitors with the ABCG2 multidrug transporter. Mol Pharmacol 65: 1485-1495, 2004.

19. Haraguchi N, Utsunomiya T, Inoue H, Tanaka F, Mimori K, Barnard GF and Mori M: Characterization of a side population of cancer cells from human gastrointestinal system. Stem Cells 24: 506-513, 2006.

20. Artavanis-Tsakonas S, Rand MD and Lake RJ: Notch signaling: Cell fate control and signal integration in development. Science 284: 770-776, 1999.
21. Lino MM, Merlo A and Boulay JL: Notch signaling in glioblastoma: A developmental drug target? BMC Med 8: 72, 2010.

22. Zhang XP, Zheng G, Zou L, Liu HL, Hou LH, Zhou P, Yin DD, Zheng QJ, Liang L, Zhang SZ, et al: Notch activation promotes cell proliferation and the formation of neural stem cell-like colonies in human glioma cells. Mol Cell Biochem 307: 101-108, 2008.

23. Fan X, Khaki L, Zhu TS, Soules ME, Talsma CE, Gul N, Koh C, Zhang J, Li YM, Maciaczyk J, et al: NOTCH pathway blockade depletes CD133-positive glioblastoma cells and inhibits growth of tumor neurospheres and xenografts. Stem Cells 28: 5-16, 2010.

24. Sikandar SS, Pate KT, Anderson S, Dizon D, Edwards RA, Waterman ML and Lipkin SM: NOTCH signaling is required for formation and self-renewal of tumor-initiating cells and for repression of secretory cell differentiation in colon cancer. Cancer Res 70: 1469-1478, 2010.

25. Zhen Y, Zhao S, Li Q, Li Y and Kawamoto K: Arsenic trioxide-mediated Notch pathway inhibition depletes the cancer stem-like cell population in gliomas. Cancer Lett 292: 64-72, 2010 . 